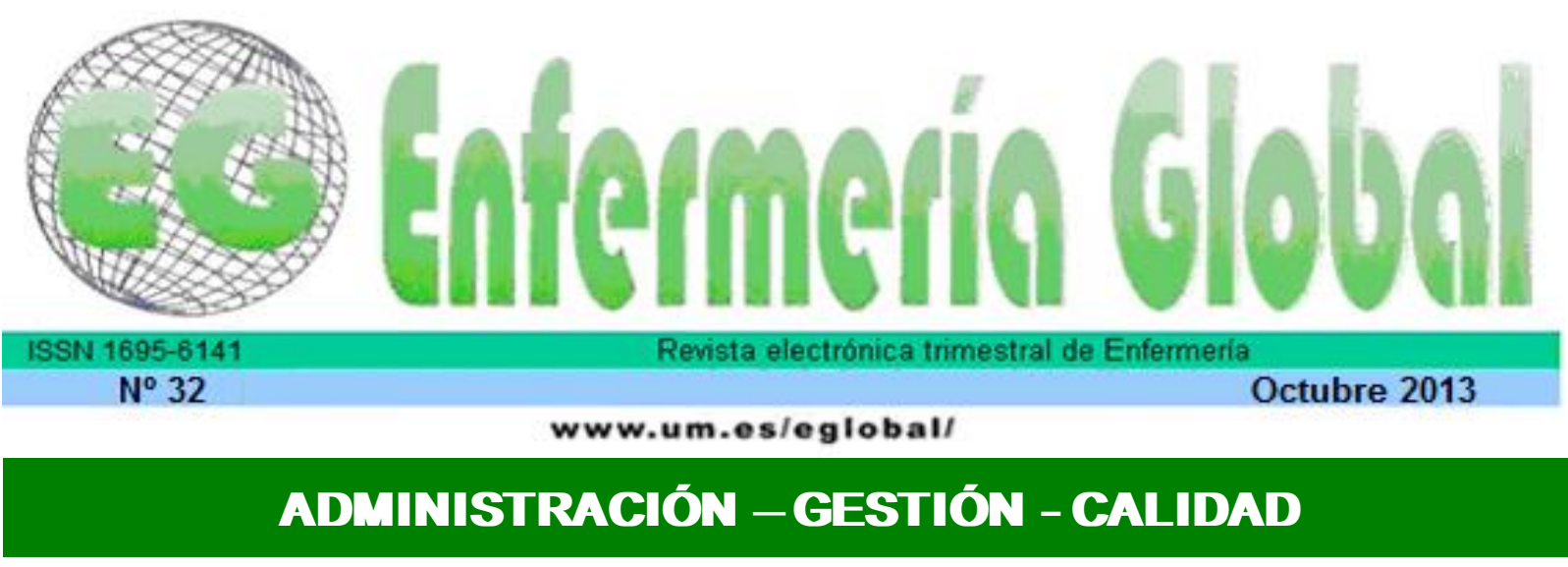

\title{
Prevalencia de errores en la utilización de medicamentos en pacientes de alto riesgo farmacológico y análisis de sus potenciales causas en una entidad hospitalaria
}

Prevalence of mistakes in the utilization of medicines in patients of high pharmacological risk and analysis of his potential reasons in a hospitable entity

\section{${ }^{*}$ Rivera Romero, Nathaly ${ }^{* *}$ Moreno de Santacruz, Rocío ${ }^{* * *}$ Escobar Espinosa, Sandra Beatriz}

"Enfermera, Joven Investigador Colciencias convocatoria 525 -. E-mail: $\frac{\text { nrivera@fucsalud.edu.co }}{{ }^{* *} \text { Enfermera, Especialista en Gestión de la Salud }{ }^{* * * *} \text { Médico candidato a Magister en Efectividad }}$
Clínica. Fundación Universitaria Ciencias de la Salud. Sede Centro - Hospital de San José de Bogotá.
Colombia.

(El presente trabajo forma parte del Proyecto financiado por COLCIENCIAS y la Fundación Universitaria de Ciencias de la Salud Convocatoria de Joven Investigador 525.)

Palabras clave: Prevalencia; errores de medicación; utilización de medicamentos; Enfermería; Farmacia; Médico.

Keywords: Prevalence; mistakes of medication; utilization of medicines; nurse; pharmacy; doctor.

\section{RESUMEN}

Los errores en el sistema de utilización de medicamentos (SUM) son un problema clínico que aumenta la morbimortalidad y el costo del tratamiento. El objetivo del trabajo fue detectar los errores en el SUM, establecer los factores asociados a su ocurrencia y su relación con el evento adverso, en pacientes con alto riesgo farmacológico, en una entidad hospitalaria de tercer nivel de Bogotá; en un periodo de tres meses del 2012.

Metodología: Se realizó observación directa en 318 pacientes adultos, la revisión de historias clínicas en 309 de ellos y la revisión de los reportes voluntarios de eventos adversos relacionados con medicamentos. Se utilizó la estadística descriptiva con Stata 10.

Resultados: Se encontró que la prevalencia global de error por cada 100 dosis paciente, en todo el sistema de utilización de medicamentos fue del $5 \%$ o más en el $95 \%$ de los pacientes; la falta de ronda de seguridad farmacológica con un $94.03 \%$ y el error de omisión del registro después de la administración del medicamento con un $68.62 \%$ fueron los errores, que ocuparon el primer lugar en campo y revisión de historias clínicas, respectivamente. El 1.89\% llegó a causar daño temporal al paciente. 
Conclusiones: Los turnos de la mañana y el fin de semana, la experiencia del personal de enfermería menor a 6 meses, y la estancia hospitalaria mayor a 10 días fueron circunstancias en donde la prevalencia de errores fue superior. La enfermera(o) tiene la responsabilidad de controlar el desarrollo de cada fase del SUM. y de liderar su mejora continua.

\section{ABSTRACT}

The mistakes in the system of utilization of medicines (SUM) are a clinical problem that increases the morbidity - mortality in the hospitals and the cost of the treatment. The aim of the work was to detect the mistakes in the SUM, to establish the factors associated to his occurrence and his relation with the adverse event, in patients hospitalized with high pharmacological risk, in a hospital of the third level of Bogota; in a period of three months of the year 2012.

Method: Direct observation was realized in 318 adults, the review of their clinical records in 309 of them and the review of the voluntary reports of adverse events related to medicines. The analysis of results was realized across descriptive statistics by Stata 10 .

Results: Thinking that the global prevalence of mistake for every 100 patient doses, in the whole system of utilization of medicines was $5 \%$ or more in $95 \%$ of the patients; the lack of round of pharmacological safety with $94.03 \%$ and the omission mistake of the record after the administration of the medicine with $68.62 \%$ were the observed mistakes, which occupied the first place in field and review of clinical histories respectively. Of the total of analyzed mistakes, $1.89 \%$ managed to damage the patient.

Conclusions: The shifts in the morning and the weekend, the experience of the personnel of minor infirmary to 6 months, and the stay at hospital longer than 10 days were circumstances where the prevalence of mistakes was high. The nurse has the responsibility of controlling de development of each period of the SUM and to lead its continuous improvement.

\section{INTRODUCCIÓN}

Los problemas relacionados con la utilización de medicamentos (PRUM) representan la mayor participación en los eventos adversos que se originan durante la atención médica hospitalaria ${ }^{(1)}$. En Colombia, se establece que estos corresponden a causas prevenibles de problemas relacionados con medicamentos, asociados al SUM, (prescripción, dispensación, administración o uso por parte del paciente o cuidador), incluyendo los fallos en el sistema de utilización de medicamentos, relacionados principalmente a la ausencia en los servicios de procesos administrativos y técnicos para garantizar la existencia de los medicamentos que realmente se necesiten, acompañados de las características de efectividad, seguridad, calidad de la información y educación para su utilización correcta ${ }^{(2)}$.

El Consejo Nacional Coordinador del Registro y Prevención de los Errores de Medicación de los Estados Unidos (National Coordinating Council on Medication Error Reporting and Prevention of USA) lo define como "un acontecimiento que puede evitarse y que es causado por una utilización inadecuada de un medicamento, produciendo lesión a un paciente, mientras la medicación está bajo control de personal sanitario, paciente o consumidor" (3)

Los errores de medicación (EM) han estado presentes desde el inicio de la utilización de los medicamentos, se han detectado en todos los sistemas de salud, aún en los países llamados desarrollados, con sistemas de mayor experiencia en seguridad y vigilancia, y han sido precisamente esos sistemas los que han conocido y analizado su ocurrencia; incluyendo cálculos económicos de los daños que éstos ocasionan ${ }^{(4)}$. Desde hace cuatro décadas gran variedad de estudios a nivel mundial reportan errores en la administración de medicamentos, los cuales representan eventos inaceptables 
para los sujetos de cuidado (personal, familiar, comunidad) porque producen complicaciones y en algunos casos hasta la muerte del paciente, generando un sobrecosto asistencial, con un tiempo de hospitalización más prolongado y por ende la pérdida de confianza en las instituciones y personal sanitario ${ }^{(4,5)}$. Los errores de medicación se han clasificado en función de su naturaleza, la clasificación de Hynniman y col. realizada en 1970, los caracteriza en tres grupos esenciales: errores de omisión, errores de comisión y discrepancias; y está la clasificación realizada por la American Society of Health-System Pharmacists en 1993 que se basó en las 12 categorías definidas en la clasificación de Barker y col ${ }^{(3)}$. Estos errores pueden presentarse debido a fallas activas por la práctica de los diferentes profesionales involucrados o a fallas latentes en el sistema de utilización de los medicamentos, bien sea en la prescripción médica, en la dispensación por farmacia o en la administración y monitorización que realiza el profesional de enfermería ${ }^{(6)}$

Es fundamental establecer la causa del error en forma no punitiva, pero sí responsable y efectiva, debido a que si bien es cierto que los efectos adversos en el paciente no son muchas veces prevenibles, existen múltiples factores de riesgo que ocurren en las diferentes disciplinas involucradas en el uso de medicamentos, que generan eventos adversos que sí se pueden controlar. Estudios realizados en otros países demuestran que estos factores están relacionados con las condiciones laborales, recursos insuficientes, nivel de preparación, años de experiencia, sobrecarga laboral, políticas institucionales y la práctica rutinaria durante su manejo, que favorecen la ocurrencia de los errores de medicamentos. ${ }^{(6,7,8)}$

Desde el componente ético, legal y disciplinar, el profesional de enfermería es el responsable de administrar el cuidado al paciente, incluyendo su participación activa en el SUM, observando y analizando sistemáticamente al paciente, las incompatibilidades y posibles reacciones farmacológicas no deseadas, pero también evaluando los procesos administrativos que facilitan o entorpecen la correcta utilización de los medicamentos; es así, como el método observacional del sistema es válido y los datos obtenidos pueden utilizarse para establecer medidas efectivas de prevención de errores ${ }^{(4,7)}$

Por otra parte, todas las repercusiones que traen los PRUM en la seguridad del paciente, en la calidad de vida laboral de los profesionales y en la buena imagen de las instituciones ante los diferentes actores del Sistema de salud, hacen necesario crear estrategias que permitan fortalecer las medidas preventivas y los programas de farmacovigilancia, a través de la notificación voluntaria de errores y la auditoría interna; generando información valiosa para la toma de decisiones institucionales, que reduzcan la frecuencia y la severidad de los errores en la utilización de medicamentos. Este panorama ha propiciado la necesidad de la gestión de riesgos en los hospitales, bajo la iniciativa de prevención de errores, donde el pilar fundamental es el apoyo de las directivas de la institución, a fin de disponer de la infraestructura y recursos necesarios para establecer una cultura de seguridad, incluyendo la formación del personal, el análisis de todo el SUM, estandarizar métodos que permitan detectar los EM y analizar las causas, con el fin de identificar los puntos críticos en la planeación de las prácticas. ${ }^{(7,9)}$

Además, desde la normatividad legal colombiana, el Sistema Obligatorio de Garantía de la Calidad en Salud, donde sus componentes de habilitación y acreditación centran su atención en la seguridad del paciente, basada en aspectos como: la competencia del personal sanitario, definición o adopción de guías y protocolos, uso racional de medicamentos, entre otros; determinando también como componente de este sistema 
el plan de auditoría de mejoramiento continuo (PAMEC) que obliga a las instituciones a estar en permanente búsqueda de sus oportunidades de mejora, para optimizar la calidad de atención prestada a sus usuarios ${ }^{(10)}$.

\section{METODOLOGÍA}

Estudio de corte transversal, con componente analítico, donde la unidad de observación es el sistema de utilización de medicamentos (SUM), para identificar los errores ocurridos en los pacientes con factores de alto riesgo farmacológico. Sobre todos los errores de medicación que se detectaron se realizó el análisis de los posibles factores asociados, y se determinó si hubo o no daño en el paciente, y en los casos positivos la magnitud del mismo.

La población está constituida por todos los pacientes adultos hospitalizados en el área médico-quirúrgica, durante el periodo de 16 abril a 15 de julio del 2012, con alto riesgo farmacológico de acuerdo a la escala de la institución, la cual determina este riesgo por medio de la valoración de las siguiente condiciones clínicas del paciente: si el paciente tiene prescripción de cinco o más medicamentos, o su tratamiento incluye antibióticos de amplio espectro o con medicamentos de ventana terapéutica estrecha y/o que tenga antecedentes de alergia medicamentosa. Se da un punto por cada ítem positivo, considerándose de alto riesgo la presencia de por lo menos un factor.

Como se incluyeron todos los pacientes que presentaron este riesgo, no se calculó muestra.

Los métodos que se utilizaron para la recolección de datos fueron:

Observación directa: aplicando instrumento de verificación de los 11 correctos establecido en el protocolo de administración de medicamentos de la institución. Se hizo solo una verificación en campo por paciente. Se distribuyó en porcentajes equivalentes en cada turno (mañana $25 \%$, tarde $25 \%$, noche $25 \%$ y fin de semana $25 \%$ ) para establecer posibles asociaciones dependientes de la dinámica que se genera en el proceso de atención del hospital en los diferentes turnos.

Revisión de historia clínica: revisión de las prescripciones médicas y el registro de administración de medicamentos durante la hospitalización, de los pacientes evaluados en la observación de campo.

Notificación voluntaria anónima o identificada: mediante el sistema de notificación de eventos adversos, se seleccionaron los casos relacionados con los medicamentos de los pacientes que cumplieron con los criterios de inclusión.

\section{RESULTADOS}

Durante el periodo de recolección de datos fueron vistos 318 pacientes y se realizó revisión a 309 historias correspondientes a los pacientes observados, lo que deduce una pérdida de información de los registros de 9 Historias clínicas (2.8\%), a las cuales no se pudo acceder en varias oportunidades. En cuanto a la revisión de los casos de reporte de evento adverso relacionado con medicamentos, ningún paciente cumplió los criterios de inclusión, en contraste, se encontraron 4 casos de pacientes con evento adverso durante el estudio, los cuales no fueron reportados a auditoría interna programa de farmacovigilancia.

La mayor cantidad de pacientes con alto riesgo medicamentoso se encontraron entre los 18 y 45 años (37,11\%), seguido de los pacientes entre 61 y 74 años (23,90\%). En 
el $25,79 \%$ de los pacientes se presentaba al menos una comorbilidad y la tendencia es de estancias hospitalarias prolongadas, dado que en el $42,14 \%$ presentó una estancia superior a 10 días.

De la población analizada el 50.94\% fueron mujeres. La especialidad más frecuente fue cirugía general $(28,93 \%)$, seguida de medicina interna $(20,44 \%)$. Las patologías fueron variadas encontrándose 177 diagnósticos principales, siendo la apendicitis la patología más frecuente con 5.35\% de los casos, seguido de la infección urinaria con el $5.03 \%$. En promedio los pacientes incluidos para la observación del SUM, tenían 10.66 medicamentos.

\section{Errores detectados en observación de campo}

La mediana del total de errores evidenciados en campo en todo el sistema de utilización de medicamentos fue de 5 , es decir el $50 \%$ de las observaciones se presentaron 5 errores o menos por paciente, distribuidos así:

En el $50 \%$ de las observaciones se encontró un error en prescripción (mediana de 1). En la tabla 1 se detalla la frecuencia de cada error y el porcentaje de afectación sobre quienes aplica. La dinámica del SUM en la dispensación de medicamentos no facilitó el seguimiento de este paso del sistema en cada uno de los pacientes. La tabla 2 refleja los ítems y la frecuencia de observación en cada uno de ellos. En el $50 \%$ de las observaciones se evidenciaron 4 errores por paciente 0 menos durante la administración de los medicamentos (mediana de 4). En la tabla 3 se detalla la frecuencia de cada error y el porcentaje de afectación sobre quienes aplica.

Tabla I: Errores observados en la prescripción de medicamentos en una entidad de salud de tercer nivel de Bogotá , 16 de abril a 15 de julio 2012. Total de observaciones por ítem 318

\begin{tabular}{|c|c|c|c|c|c|}
\hline Ítem evaluado & No aplica & $\begin{array}{c}\text { No } \\
\text { cumple }\end{array}$ & Cumple & $\begin{array}{l}\text { Total de } \\
\text { pacientes } \\
\text { en quienes } \\
\text { aplica }\end{array}$ & $\begin{array}{l}\% \text { Error en el } \\
\text { total de los } \\
\text { pacientes en } \\
\text { quienes } \\
\text { aplica }\end{array}$ \\
\hline Firma y Sello - Legible & 0 & 130 & 188 & 318 & $40,88 \%$ \\
\hline La orden medica es legible & 0 & 115 & 203 & 318 & $36,16 \%$ \\
\hline $\begin{array}{l}\text { Realiza formulario de no POS o } \\
\text { de medicamentos controlados } \\
\text { oportunamente }\end{array}$ & 293 & 7 & 18 & 25 & $28,00 \%$ \\
\hline Uso de nombre genérico & 0 & 10 & 308 & 318 & $3,14 \%$ \\
\hline Dosis & 0 & 6 & 312 & 318 & $1,89 \%$ \\
\hline Horario & 0 & 6 & 312 & 318 & $1,89 \%$ \\
\hline Medicamento correcto & 0 & 4 & 314 & 318 & $1,26 \%$ \\
\hline Dosis correcta & 0 & 4 & 314 & 318 & $1,26 \%$ \\
\hline Vía de administración & 0 & 3 & 315 & 318 & $0,94 \%$ \\
\hline
\end{tabular}


Tabla II: Errores observados en la dispensación de medicamentos, en una entidad de salud de tercer nivel de Bogotá ,16 de abril a 15 de julio 2012.

\begin{tabular}{|c|c|c|c|c|c|}
\hline Ítem evaluado & $\begin{array}{c}\text { No } \\
\text { aplica }\end{array}$ & $\begin{array}{c}\text { No } \\
\text { cumple }\end{array}$ & Cumple & $\begin{array}{c}\text { Total de } \\
\text { pacientes } \\
\text { en } \\
\text { quienes } \\
\text { aplica }\end{array}$ & $\begin{array}{c}\% \text { Error en } \\
\text { el total de } \\
\text { los } \\
\text { pacientes en } \\
\text { quienes } \\
\text { aplica }\end{array}$ \\
\hline $\begin{array}{l}\text { Ante prescripción } \\
\text { dudosa confirma } \\
\text { directamente con el } \\
\text { médico }\end{array}$ & 313 & 2 & 3 & 5 & $40,00 \%$ \\
\hline $\begin{array}{l}\text { Utiliza etiquetas } \\
\text { adicionales }\end{array}$ & 111 & 56 & 151 & 207 & $27,05 \%$ \\
\hline $\begin{array}{l}\text { Hace entregas } \\
\text { completas }\end{array}$ & 235 & 22 & 61 & 83 & $26,51 \%$ \\
\hline $\begin{array}{l}\text { En horario } \\
\text { establecido }\end{array}$ & 232 & 18 & 68 & 86 & $20,93 \%$ \\
\hline Nombre Genérico & 0 & 8 & 310 & 318 & $2,52 \%$ \\
\hline $\begin{array}{l}\text { Dispensación del } \\
\text { Medicamento }\end{array}$ & 233 & 2 & 83 & 85 & $2,35 \%$ \\
\hline Entrega medicamento & 232 & 2 & 84 & 86 & $2,33 \%$ \\
\hline Dosis & 0 & 7 & 311 & 318 & $2,20 \%$ \\
\hline $\begin{array}{l}\text { Fecha de } \\
\text { Vencimiento }\end{array}$ & 0 & 7 & 311 & 318 & $2,20 \%$ \\
\hline Etiquetado Correcto & 0 & 6 & 312 & 318 & $1,89 \%$ \\
\hline Correcto & 235 & 1 & 82 & 83 & $1,20 \%$ \\
\hline $\begin{array}{l}\text { Entrega el } \\
\text { medicamento con } \\
\text { orden Medica }\end{array}$ & 234 & 1 & 83 & 84 & $1,19 \%$ \\
\hline $\begin{array}{l}\text { Evita el envasado y } \\
\text { dispensación de } \\
\text { medicamentos en } \\
\text { multidosis. }\end{array}$ & 261 & 0 & 57 & 57 & $0,00 \%$ \\
\hline
\end{tabular}


Tabla III: Errores observados en la administración de medicamentos, en una entidad de salud de tercer nivel de Bogotá ,16 de abril a 15 de julio 2012. Total de observaciones por ítem 318

\begin{tabular}{|c|c|c|c|c|c|}
\hline Ítem evaluado & $\begin{array}{c}\text { No } \\
\text { aplica }\end{array}$ & Cumple & $\begin{array}{l}\text { No } \\
\text { cumple }\end{array}$ & $\begin{array}{c}\text { Total de } \\
\text { pacientes } \\
\text { en } \\
\text { quienes } \\
\text { aplica }\end{array}$ & $\begin{array}{l}\% \text { Error en } \\
\text { el total de } \\
\text { los } \\
\text { pacientes } \\
\text { en quienes } \\
\text { aplica }\end{array}$ \\
\hline $\begin{array}{l}\text { Realiza ronda de } \\
\text { seguridad. }\end{array}$ & 0 & 19 & 299 & 318 & $94,03 \%$ \\
\hline $\begin{array}{l}\text { Lava el equipo con } 20 \text { cc } \\
\text { después de administrar } \\
\text { medicamento IV }\end{array}$ & 105 & 21 & 192 & 213 & $90,14 \%$ \\
\hline $\begin{array}{l}\text { Ubica en la portada de la } \\
\text { historia clínica, hoja de } \\
\text { medicamentos y manilla, } \\
\text { las alergias } \\
\text { farmacológicas del } \\
\text { paciente. }\end{array}$ & 243 & 19 & 56 & 75 & $74,67 \%$ \\
\hline Realiza lavado de manos & 0 & 165 & 153 & 318 & $48,11 \%$ \\
\hline $\begin{array}{l}\text { Registra y firma la hoja de } \\
\text { medicamentos después } \\
\text { de administrar el } \\
\text { medicamento. }\end{array}$ & 0 & 187 & 131 & 318 & $41,19 \%$ \\
\hline $\begin{array}{l}\text { Justifica en la hoja de } \\
\text { evolución de enfermería } \\
\text { el motivo por el cual no } \\
\text { administra un } \\
\text { medicamento }\end{array}$ & 300 & 12 & 6 & 18 & $33,33 \%$ \\
\hline $\begin{array}{l}\text { Evita el uso de siglas } \mathrm{NH} \text {, } \\
\text { NF en las casillas de } \\
\text { medicamentos. Verifica } \\
\text { inconsistencias y reporta } \\
\text { a la coordinadora. }\end{array}$ & 297 & 15 & 6 & 21 & $28,57 \%$ \\
\hline $\begin{array}{l}\text { Si existe alguna duda no } \\
\text { administra, y consulta. }\end{array}$ & 294 & 18 & 6 & 24 & $25,00 \%$ \\
\hline $\begin{array}{l}\text { Justifica la administración } \\
\text { de un medicamento } \\
\text { "según necesidades" }\end{array}$ & 309 & 7 & 2 & 9 & $22,22 \%$ \\
\hline $\begin{array}{l}\text { En medicamentos por vía } \\
\text { IV, verifica permeabilidad } \\
\text { de vena }\end{array}$ & 105 & 175 & 38 & 213 & $17,84 \%$ \\
\hline
\end{tabular}




\begin{tabular}{|c|c|c|c|c|c|}
\hline Usa tapabocas & 0 & 266 & 52 & 318 & $16,35 \%$ \\
\hline $\begin{array}{l}\text { Siempre que una dosis } \\
\text { prescrita parece } \\
\text { inadecuada comprueba } \\
\text { de nuevo }\end{array}$ & 262 & 47 & 9 & 56 & $16,07 \%$ \\
\hline $\begin{array}{l}\text { Evita el uso joyas en las } \\
\text { manos }\end{array}$ & 0 & 271 & 47 & 318 & $14,78 \%$ \\
\hline Verifica la orden medica & 0 & 281 & 37 & 318 & $11,64 \%$ \\
\hline $\begin{array}{l}\text { Investiga si el paciente } \\
\text { padece alergias y } \\
\text { descarta interacción } \\
\text { farmacológica. }\end{array}$ & 0 & 281 & 37 & 318 & $11,64 \%$ \\
\hline $\begin{array}{l}\text { Desecha cualquier forma } \\
\text { farmacéutica que no esté } \\
\text { correctamente } \\
\text { identificada. }\end{array}$ & 309 & 8 & 1 & 9 & $11,11 \%$ \\
\hline $\begin{array}{l}\text { Administra medicamento } \\
\text { a la hora correcta }\end{array}$ & 0 & 283 & 35 & 318 & $11,01 \%$ \\
\hline $\begin{array}{l}\text { Verifica la reconciliación } \\
\text { medicamentosa. }\end{array}$ & 0 & 283 & 35 & 318 & $11,01 \%$ \\
\hline $\begin{array}{l}\text { Planea los medicamentos } \\
\text { de acuerdo a los } \\
\text { sinergismos, } \\
\text { antagonismos, ultima } \\
\text { dosis recibida por el } \\
\text { paciente. }\end{array}$ & 0 & 285 & 33 & 318 & $10,38 \%$ \\
\hline $\begin{array}{l}\text { Comprueba que el } \\
\text { paciente no toma ningún } \\
\text { medicamento ajeno al } \\
\text { prescrito }\end{array}$ & 0 & 286 & 32 & 318 & $10,06 \%$ \\
\hline $\begin{array}{l}\text { Si la vía no parece en la } \\
\text { prescripción consulta }\end{array}$ & 243 & 70 & 5 & 75 & $6,67 \%$ \\
\hline $\begin{array}{l}\text { Tiene en cuenta las dosis } \\
\text { máximas y mínimas de } \\
\text { acuerdo al paciente y } \\
\text { condiciones clínicas }\end{array}$ & 0 & 302 & 16 & 318 & $5,03 \%$ \\
\hline $\begin{array}{l}\text { Administra la dosis } \\
\text { correcta. }\end{array}$ & 0 & 305 & 13 & 318 & $4,09 \%$ \\
\hline $\begin{array}{l}\text { Se asegura que la vía de } \\
\text { administración es la } \\
\text { correcta }\end{array}$ & 0 & 305 & 13 & 318 & $4,09 \%$ \\
\hline
\end{tabular}




\begin{tabular}{|c|c|c|c|c|c|}
\hline $\begin{array}{l}\text { Administra el } \\
\text { medicamento correcto }\end{array}$ & 0 & 310 & 8 & 318 & $2,52 \%$ \\
\hline $\begin{array}{l}\text { Comprueba el nombredel } \\
\text { paciente al preparar el } \\
\text { medicamento }\end{array}$ & 0 & 310 & 8 & 318 & $2,52 \%$ \\
\hline $\begin{array}{l}\text { Informa e instruye al } \\
\text { paciente sobre el } \\
\text { medicamento que será } \\
\text { administrado. }\end{array}$ & 0 & 310 & 8 & 318 & $2,52 \%$ \\
\hline $\begin{array}{l}\text { Identifica el medicamento } \\
\text { correcto y comprueba la } \\
\text { fecha de caducidad del } \\
\text { mismo. }\end{array}$ & 0 & 311 & 7 & 318 & $2,20 \%$ \\
\hline $\begin{array}{l}\text { Comprueba el nombre del } \\
\text { paciente al administrar el } \\
\text { medicamento. }\end{array}$ & 0 & 311 & 7 & 318 & $2,20 \%$ \\
\hline $\begin{array}{l}\text { Indica al paciente nombre } \\
\text { del medicamento y su } \\
\text { acción. }\end{array}$ & 0 & 311 & 7 & 318 & $2,20 \%$ \\
\hline $\begin{array}{l}\text { Inicia mezclas especiales, } \\
\text { antibióticos y analgesia } \\
\text { con un tiempo no mayor a } \\
2 \text { horas de formulación. }\end{array}$ & 45 & 267 & 6 & 273 & $2,20 \%$ \\
\hline $\begin{array}{l}\text { Administra el } \\
\text { medicamento por la vía } \\
\text { correcta }\end{array}$ & 0 & 312 & 6 & 318 & $1,89 \%$ \\
\hline $\begin{array}{l}\text { Registra todos los } \\
\text { medicamentos } \\
\text { administrados. }\end{array}$ & 0 & 312 & 6 & 318 & $1,89 \%$ \\
\hline Mantiene cabello recogido & 0 & 312 & 6 & 318 & $1,89 \%$ \\
\hline $\begin{array}{l}\text { Administra el } \\
\text { medicamento al paciente } \\
\text { indicado. }\end{array}$ & 0 & 313 & 5 & 318 & $1,57 \%$ \\
\hline $\begin{array}{l}\text { Comprueba la identificació } \\
\text { del paciente, preguntando } \\
\text { nombres y apellidos } \\
\text { completos, fecha de } \\
\text { nacimiento y documento de } \\
\text { identidad. }\end{array}$ & 0 & 313 & 5 & 318 & $1,57 \%$ \\
\hline $\begin{array}{l}\text { Antes de preparar y admini: } \\
\text { un medicamento tiene en cı } \\
\text { la técnica aséptica correcta }\end{array}$ & 0 & 314 & 4 & 318 & $1,26 \%$ \\
\hline
\end{tabular}




\section{Errores detectados durante la revisión de historias clínicas}

\section{Análisis Univariado}

La mediana del total de errores evidenciados en la prescripción de medicamentos fue de 2 , es decir en el $50 \%$ de las observaciones se encontraron 2 errores o menos por paciente. La mediana de prevalencia de error del personal médico por cada 100 dosis administradas fue de $3.58 \%$.

La mediana del total de errores observados en la historia clínica, en lo referente al registro de la administración de medicamentos que debe realizar enfermería en el SUM, fue de 5 , es decir el $50 \%$ de las observaciones se encontraron 5 errores 0 menos por paciente. La mediana de prevalencia de error de enfermería por cada 100 dosis administradas fue de $9.74 \%$, es decir, en el $50 \%$ de las observaciones, se encontró una prevalencia de $9.9 \%$ o menos por cada 100 dosis paciente.

Durante la revisión de historias clínicas, los errores evidenciados de dispensación se detectaron gracias al registro específico que realiza en algunas ocasiones enfermería, como por ejemplo la no disponibilidad del medicamento, dado que el servicio de farmacia, no realiza registros en las historias clínicas.

\section{Tipos y severidad de errores detectados}

El error más frecuente identificado durante la revisión de historias clínicas fue error por omisión, representando el $68.62 \%$ de los errores encontrados, en donde el $50 \%$ de los pacientes presentaron 4 errores o menos (mediana de 4), seguido por errores en prescripción con un porcentaje del $24,90 \%$ de los errores encontrados. Se observa que el $4,29 \%$ presenta errores en el horario de prescripción. En cuanto a error por medicamento no autorizado, error en la técnica o dosis extras se observa un porcentaje menor al $2 \%$ y no se identificaron errores en dosis, error en la vía 0 paciente equivocado, por parte de enfermería. En la severidad de los EM detectados, en el $89,31 \%$ se observó que el error alcanzó al paciente pero no causó daño, mientras en el 1,89\% ocurrió un error que alcanzó al paciente y precisó monitorización y/o intervención para comprobar que no había sufrido daño, en el 0,31\% ocurrió un error, teniendo la necesidad de tratamiento, y causó un daño temporal al paciente. En el $3.46 \%$ de la muestra no se obtuvieron datos de la severidad del error.

\section{Análisis Bivariado}

\section{Total de comorbilidades vs error sistema, comparar medianas}

Se encuentra que en los pacientes con cero comorbilidades tienen una mediana de total de errores en el sistema de 13, la cual es menor con respecto a los pacientes con al menos una comorbilidad la cual fue de 15, sin embargo estas diferencias no se consideran estadísticamente significativas valor de $p=0.132$ prueba de wilkoxon.

\section{Estancia vs error del SUM. (Comparación de medianas)}

Se encuentra que en los pacientes con estancia menor a 10 días tienen una mediana de total de errores en el sistema de 17.5, la cual es menor con respecto al pacientes con una estancia mayor a 10 días, la cual fue de 23 , estas diferencias fueron estadísticamente significativas valor de $p=0.00$, prueba de wilkoxon; lo que demuestra que a mayor estancia, mayor posibilidad de error. 


\section{Edad vs error del sistema. (Comparación de medianas)}

Se comparó si existen diferencias en el total de errores encontrados entre los pacientes con mayor edad, encontrando que aunque la mediana de errores es mayor en los pacientes mayores de 65 años, esta diferencia no alcanza a ser estadísticamente significativa valor de $\mathrm{p}=0.40$, prueba de wilkoxon.

\section{Experiencia de la enfermera vs error del SUM. (Comparación de medianas)}

En cuanto a la experiencia, el personal de enfermería con menos de 6 meses de práctica tuvo una mediana de errores mayor (14) respecto a las que tenían 6 meses o más (7.5) $p=0.01$, prueba de wilkoxon. Lo que demuestra que a menor experiencia mayor posibilidad de error.

\section{DISCUSIÓN}

La complejidad del sistema de atención hospitalaria, el déficit de recursos en salud, la inestabilidad del personal y el aumento de la expectativa de vida y de las enfermedades crónicas, representan un reto para todos los actores involucrados en el sistema de utilización de medicamentos ${ }^{(11)}$. Los resultados obtenidos reflejan que hay condiciones de riesgo susceptibles de control, pero que deben de soportarse en la voluntad política de la institución de crear sistemas simples, integrales, evaluables y costo efectivos, que favorezcan la optimización de la seguridad del paciente y la efectividad clínica del tratamiento farmacológico ${ }^{(6)}$.

Dentro de las fallas propias del proceso desarrollado en la institución, se encontró en la revisión de las historias el error por omisión del registro que realiza enfermería como el más frecuente $(68.62 \%)$, seguido del de prescripción (24.3\%); en otros estudios las distribuciones de los errores son distintas: dosis (24\%), horario (22.9\%) 0 medicamentos no autorizados (13.5\%) (12); horario (68.7\%), omisión (12.6\%), dosis equivocada (12.4\%) (13). En campo los tres principales hallazgos fueron: la no realización de la ronda de seguridad una vez aplicado el medicamento por parte de enfermería (94,03\%), no lavar el equipo de venoclisis con 20 cc de solución salina, después de la administración de medicamentos vía endovenosa $(90,14 \%)$ y la no señalización de las alergias farmacológicas del paciente en la portada de la historia clínica, manilla y hoja de medicamentos, $(74,67 \%)$, aspectos que son relevantes en la seguridad clínica del paciente por su riesgo potencial de causar eventos adversos, por lo tanto sus causas deben analizarse metodológicamente y priorizarse en los planes de mejora continua de enfermería. ${ }^{(9)}$

La proporción de errores que llegó a causar daño significativo al paciente fue del $1.89 \%$ y en todos los casos se pudo resolver sin dejar secuelas; sin embargo la tasa reportada por la literatura es superior $(7.5 \%$ al $10.4 \%)(14,15,16,17)$, lo que puede estar relacionado con la debilidad de la cultura del reporte de eventos adversos, o como lo menciona Martín Muñoz, Begoña en su estudio ${ }^{(18)}$, puede deberse a la falta de capacitación sobre la clasificación de los errores de medicación.

En el estudio realizado en 2011 por la enfermera Diana Yamile Ramos Castro, se utilizó la escala validada de la frecuencia de las diez distracciones planteadas por la autora de la Lista de Chequeo: Theresa Pape, relacionadas con las variables de contexto: turnos, área de hospitalización, horarios de programación de medicamentos, tiempo transcurrido en el proceso de administración de medicamentos y número de pacientes asignados a cada profesional de enfermería; encontrando que las distracciones que con mayor frecuencia se presentaron fueron en su orden: miembros 
del personal, conversaciones, llamadas telefónicas y médico, representando el $83,38 \%$., pero no reporta la asociación de los errores con los turnos ${ }^{(19)}$.

En contraste con los hallazgos del presente estudio, se encontró que los turnos con más errores fueron los de las mañanas y los fines de semana; datos interesantes para realizar otro estudio donde se aplique esta escala para analizar si la experiencia de las enfermeras menor a 6 meses, para administrar un servicio de hospitalización asignadas al turno de la mañana, en el cual se identificó en la institución mayor número de actividades por dirigir y ejecutar, está incidiendo en forma directa, en la mayor ocurrencia de los errores. Respecto a los fines de semana, puede estar afectando la forma de su programación, ya que de acuerdo a la revisión de las planillas de asignación de turnos de la institución la enfermera no tiene ningún día de descanso previo a su turno de fin de semana, cuya jornada laboral es de 12 a 18 horas. Estudios como estos invitan a las directivas de enfermería, a revisar las actividades propias de cada turno, incluyendo los horarios de programación de medicamentos y la programación de turnos de fin de semana.

Los pacientes con estancia superior a 10 días y los atendidos por enfermeras con experiencia menor a 6 meses presentaron mayor de cantidad de errores, lo que refleja la necesidad de fortalecer el proceso de inducción, enfatizando en la capacitación de las condiciones en que se deben de cumplir las actividades de cada fase del SUM, la capacitación en la identificación de errores de medicación y la sensibilización hacia la cultura del reporte de los incidentes y eventos adversos. Es necesario también impulsar el autocontrol estableciendo sistemas de auditora interna en cada servicio, para identificar oportunamente las fallas en el sistema y actuar sobre ellas, como por ejemplo, la inclusión del registro de medicamentos en el recibo de turno, para controlar el error de omisión del registro ${ }^{(7)}$.

Por otro lado, la caracterización de los pacientes muestra un grupo bastante heterogéneo, donde no predomina una especialidad o patología en particular, llamando la atención que varios pacientes cumplieron el criterio de alto riesgo farmacológico por haber ingresado al hospital por el servicio de urgencias, donde se les formula - por costumbre - medicamentos como ranitidina y metoclopramida. La edad y la presencia de comorbilidades no mostraron diferencias significativas en cuanto a la frecuencia de errores, en otro estudio realizado en población pediátrica se encontró un hallazgo similar ${ }^{(20)}$.

\section{CONCLUSIONES}

La prevalencia de errores en el SUM, para los pacientes de alto riesgo farmacológico, sugiere una mayor dificultad en el manejo de los medicamentos en este tipo de pacientes y deja en evidencia que los procesos que apoyan al SUM no se realizan de manera estandarizada. Los turnos del fin de semana y las mañanas, la experiencia de las enfermeras, y la estancia hospitalaria prolongada, fueron factores relacionados con mayor prevalencia de errores.

El programa de farmacovigilancia debe tener como pilares estratégicos, incentivar la cultura no punitiva pero sí responsable en los actores del SUM, donde se identifique el análisis de los incidentes y eventos adversos, a manera de una oportunidad para incidir en los puntos críticos.

Como institución universitaria se debe de apoyar en convenios docencia - servicio para innovar estrategias que fortalezcan la estandarización del sistema, el control oportuno del cumplimiento del protocolo institucional y la educación permanente y 
suficiente, especialmente en el personal de enfermería, quien es en últimas el profesional que participa en todas las etapas del sistema de utilización de medicamentos, sin restarle importancia al trabajo en equipo, que debe desarrollarse entre todos los involucrados en el SUM.

\section{REFERENCIAS BIBLIOGRÁFICAS}

1. Decreto por el cual se reglamenta el servicio farmacéutico y se dictan otras disposiciones, decreto 2200 de 2005, Diario Oficial 45954 de (29 de junio de 2005)

2. Otero M.J., Martin RR., Robles M.D., Codina C-, "Errores de medicación" farmaciahospitalariaSEFH.,2002,713-47 Disponible

en: http://www.ismpespana.org/ficheros/Fichero07.pdf.

3. National Coordinating Council for Medication Error Reporting and Prevention. NCCMERPTaxonomy of medication errors, 1998.Disponible http://www.nccmerp.org/aboutmederrors.htm

4. Segura, Blasco, Mariño, P, E, Saliente Aznar, Ma․ T, Yanguas E. Pol, AlósAlmiñana, M, Castells, Molina M., Velasco, Álvarez $M^{a}{ }^{a}$. L. Desarrollo de un método observacional prospectivo de estudio de Errores de Medicación para su aplicación en hospitales.Farmacia hospitalaria.Vol. 25. N. ${ }^{\circ}$ 5, pp. 253-273, 2001

5. BoletínN $N^{\circ} 19$ de farmacovigilancia Grupo de Farmacovigilancia de la subdirección de medicamentos y productos biológicos del Instituto Nacional de Vigilancia De Medicamentos y Alimentos [Internet]. 2008 Jun; Available from: http://www.invima.gov.co/portal/documents/portal/documents/root/Boletin19de2008.p $\underline{\mathrm{df}}$

6. Otero López María José. Nuevas iniciativas para mejorar la seguridad de la utilización de los medicamentos en los hospitales. Rev. Esp. Salud Pública [revista en la Internet]. 2004 Jun [citado 2013 Mar 05]; 78(3): 323-339. Disponible en: http://scielo.isciii.es/scielo.php?script=sci arttext\&pid=S1135-

$57272004000300003 \& \operatorname{lng}=\mathrm{es}$

7. Vilá-de-Muga M, Colom-Ferrer L, Gonzalez-Herrero M, Luaces-Cubells C. Factors associated with medication errors in the pediatric emergency department. Pediatric EmergCare. 2011 Abr; 27(4):290-294.

8. Sentimentos de profissionais de enfermagemapós a ocorrência de erros de medicação [Internet]. 2007 Dic [citado 2011 Jul 9];Availablefrom: http://bases.bireme.br/cgi-

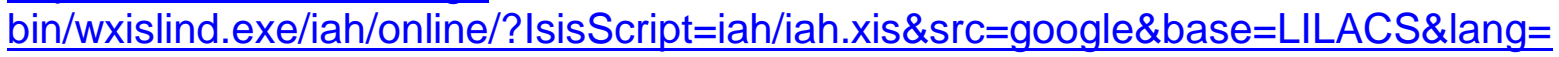
p\&nextAction=Ink\&exprSearch=471918\&indexSearch=ID

9. Estudio lbeas: prevalencia de efectos adversos en hospitales de Latinoamérica. Informe de resultados Colombia. Organización Mundial De La Salud. 2010. Disponible en:

http://mps.minproteccionsocial.gov.co/newsogc/comite/User/Library/Folders/Comite 8/IBEAS\%20FINAL.pdf

10. Decreto por el cual se establece el Sistema Obligatorio de Garantía de Calidad de la Atención de Salud del Sistema General de Seguridad Social en Salud. decreto 1011 de 2006, Diario Oficial 46230 (03 de abril de 2006).

11. ParavicKlijn, T. (2010). Enfermería y globalizacion.Cienciaenfermería, 9- 15. Disponible en: http://www.scielo.cl/scielo.php?script=sci_arttext\&pid=S071795532010000100002\&lng=es. doi: 10.4067/

12. Thalyta Cardoso, Alux Teixeira, Silvia Helena de BortoliCasani. Root Analysis: Evaluation of medication Errors at a University Hospital. Rev Esc Enfermería USP 2010, 44(1), 136-46. 
13. María Cristina Baracaldo. Detección de errores en medicación en la administración de medicamentos. Universidad Nacional de Colombia. Revista Colombiana de Ciencias Químico farmacéuticas. Vol 29: 26 - 31. Febrero de 2000.

14. AkJha, N Prasopa-Plaizer, I Larizgotia, D W Bates. On Behalf of the Research Priority Setting Working Group of the WHO World Alliance for Patient Safety. Patient safety research: an overviem of the global evidence. QualSaf Health Care 2010; 19:42-47.

15. Baker GR, Norton PG, Flintoft V, et al, The Canadian Adverse Events Study, the incidence of adverse events hospital patients in Canada. CMAJ 2004; 170: 167886.

16. Miller GC, Britth HC, Valenti L. Adverse drug events in general practice patients in Australia. Med J Aust 2006; 184:321-4.

17. Bates DW, Cullen, Laird N, et al. Incidence of adverse drug events and potential adverse drug events. Implications for prevention. ADE prevention StudyGroup. JAMA 1995; 274:29-34.

18. Martín Muñoz, Begoña. Errores de medicación y enfermería: una revisión clínica. Evidentia. 2008 sep-oct; 5(23). Disponible en: <http://www.indexf.com/evidentia/n23/ev6787.php

19. Diana Yamile Ramos Castro, Distracciones del profesional de Enfermería en el proceso de administración de medicamentos en el Hospital Universitario Fundación Santafé de Bogotá. Universidad Nacional de Colombia facultad de Enfermería Maestría en Enfermería con Énfasis en Gerencia en servicios de salud Bogotá,Colombia 2011

20. Paul E. Plsek, MS. Quality Improvement Methods in Clinical Medicine. Pediatrics Vol. 103, Jan, 1999, pp. 203-213. 